\section{Queixas osteomusculares relacionadas ao trabalho relatadas por mulheres de centro de ressocialização}

\author{
Work-related musculoskeletal complaints by \\ women in a social rehabilitation center
}

\author{
Eliane Cristina Pastre 1 \\ Guaracy Carvalho Filho 1,2 \\ Carlos Marcelo Pastre 1,3 \\ Carlos Roberto Padovani 4 \\ Josiane Schadeck de Almeida 1 \\ Jayme Netto Júnior 1,3
}

\author{
1 Programa de Pós Graduação \\ em Ciências da Saúde, \\ Faculdade de Medicina \\ de São José do Rio Preto, \\ São José do Rio Preto, Brasil. \\ 2 Departamento de Ortopedia \\ e Traumatologia, Faculdade \\ de Medicina de São José \\ do Rio Preto, São José do \\ Rio Preto, Brasil \\ ${ }^{3}$ Faculdade de Ciências e \\ Tecnologia, Universidade \\ Estadual Paulista, \\ Presidente Prudente, Brasil. \\ ${ }_{4}^{4}$ Instituto de Biociências, \\ Universidade Estadual \\ Paulista, Botucatu, Brasil. \\ Correspondência \\ E. C. Pastre \\ Programa de Pós Graduação \\ em Ciências da Saúde, \\ Faculdade de Medicina de \\ São José do Rio Preto. \\ Rua Raul de Carvalho 271, \\ São José do Rio Preto, SP \\ 15025-300, Brasil. \\ elianepastre@hotmail.com
}

\begin{abstract}
Considering work-related complaints as a public health problem, the current study aimed to analyze the frequency of musculoskeletal complaints and the association between their occurrence and severity and anthropometric and work variables. The sample included 146 women from a social rehabilitation center who answered a validated questionnaire with anthropometric data and history of work-related musculoskeletal complaints. The Goodman test was used to analyze the association between and within variables. We observed a high rate of complaints after beginning work activity (94.19\%). Subjects that required sick leave showed higher mean age and weight. The most severe complaints related to back pain. Sick leave was more common among women who had worked longer. The study concluded that there was a high frequency of work-related complaints and an association between: higher weight and height values and severity; higher age and sick leave; more serious complaints and back pain; and longer time on the job and need for sick leave.
\end{abstract}

Cumulative Trauma Disorders; Women's Health; Occupational Health; Anthropometry

\section{Introdução}

As lesões por esforços repetitivos (LER) ou distúrbios osteomusculares relacionados ao trabalho (DORT) são caracterizados por dor nos locais anatômicos mais utilizados em funções ocupacionais. Podem gerar desordens motoras, psicológicas e sociais, resultando em redução da produção e, até mesmo, no afastamento das ocupações, sendo notada elevada freqüência dentro de populações diversas, principalmente entre mulheres jovens $1,2,3,4$.

Dentre os principais fatores para sua instalação, destacam-se a intensificação da jornada de trabalho e a necessidade de aumento de produção, além da repetitividade de movimentos, manutenção de posturas inadequadas, esforço físico, invariabilidade das tarefas, pressão mecânica sobre determinados segmentos do corpo, trabalho muscular estático, impactos e vibrações 1 . Tais condições são notadas nos mais variados tipos de atividades ocupacionais e suas respectivas populações vêm sendo estudadas a fim de identificar causas e determinar intervenções $1,5,6,7$.

Contudo, não foram encontrados registros sobre o tema relacionados a reeducandas inseridas em centros de ressocialização. Esta população realiza, durante cumprimento da pena, atividades manuais ou de serviços gerais, comuns entre populações não institucionalizadas, que apresentam fatores que predispõem aos sinto- 
mas osteomusculares. Assim, bons níveis de saúde geral, incluindo as condições do sistema músculo-esquelético, é de fundamental importância, não só no sentido físico, mas também no contexto da socialização ou integração ao trabalho ${ }^{8}$.

O presente estudo justifica-se pela presença de altas taxas de sintomas osteomusculares na população feminina de maneira geral e pela possibilidade de afastamento de atividades ocupacionais das acometidas, caracterizandose, segundo Pereira 9, como problema de saúde pública, por sua abrangência, transcendência e vulnerabilidade.

Cabe destacar que, além da escassez de estudos sobre problemas de saúde das mulheres em prisões brasileiras, o encarceramento parece apresentar uma oportunidade singular, não só pelo controle de variáveis pela sistematização das atividades diárias, mas também pela possibilidade de implementação de programas terapêuticos, medidas preventivas e ações educativas para a saúde das mulheres institucionalizadas 10 .

Nesse sentido, foi definido como objetivo do estudo descrever a freqüência das queixas osteomusculares e associar sua ocorrência e severidade às variáveis antropométricas e às características do trabalho de reeducandas inseridas em centro de ressocialização.

\section{Métodos}

\section{Identificação do tipo de estudo e população}

O estudo é caracterizado como sendo do tipo observacional, analítico e transversal com componente retroanalítico, como descrito por Pereira 9.

A população encarcerada, no momento da pesquisa, era composta por 200 mulheres. Foram incluídas neste estudo, para análise e associação de variáveis, mulheres que realizavam alguma atividade laboral, com idade a partir dos 18 anos, sem distinção de etnia, e que apresentavam sinais ou sintomas osteomusculares relacionados ao trabalho, iniciados durante $o$ período de reclusão. A casuística do estudo foi composta por 146 mulheres reeducandas do Centro de Ressocialização Feminino de São José do Rio Preto, São Paulo, Brasil. As médias observadas foram 31,3 anos de idade (DP $=9,8$ ); $71,0 \mathrm{~kg}$ de peso $(\mathrm{DP}=15,6) ; 160,2 \mathrm{~cm}$ de estatura $(\mathrm{DP}=6,5)$ e 7,4 meses de tempo de reclusão e trabalho $(\mathrm{DP}=4,5)$.

Foram excluídas 54 detentas, das quais 45 apresentavam sinais e sintomas músculo-esqueléticos anteriores às atividades laborais realizadas na prisão. As queixas eram referentes a acidentes de trânsito ou domésticos $(n=20)$, doen- ças osteomusculares relacionadas ao trabalho (n $=17$ ) e cirurgias $(n=8)$. Além disso, não referiram quaisquer queixas relacionadas ao trabalho um total de nove mulheres.

\section{Procedimentos de coleta e descrição do questionário}

Os dados foram coletados por meio de um questionário aplicado pela própria pesquisadora, nos respectivos locais de trabalho, durante o período de intervalo das atividades das participantes da pesquisa.

O instrumento de coleta apresentava dados referentes a informações pessoais, como número de identificação do paciente, idade, peso, estatura, local, tipo e tempo de trabalho.

Além disso, foi solicitada descrição das queixas osteomusculares, conforme as registradas no Nordic Musculoskeletal Questionnaire (NMQ) validado em português 6. Esse modelo é utilizado internacionalmente e foi desenvolvido para padronizar pesquisas sobre o tema, sendo de fácil compreensão, com questões simples e diretas 11,12 .

Após estudo piloto, foram inseridas questões sobre severidade da queixa, como sinais e sintomas leves, moderados e acentuados, caracterizando relação com inconveniência, modificação ou interrupção das atividades. Além disso, as regiões anatômicas, braço e panturrilha, não destacadas no questionário original foram acrescentadas, em face de sua ocorrência no estudo prévio.

Visando a facilitar a identificação do exato local da queixa, a ficha de coleta continha uma figura humana, com vista posterior, dividida em: pescoço, ombro, braço, região dorsal, cotovelo, antebraço, região lombar, punho/mão/dedo, quadril/coxa, joelho, panturrilha, tornozelo/pé. As questões relacionavam-se a cada área anatômica, referindo-se a queixas dos últimos 12 meses, necessidade de redução ou afastamento das atividades, severidade e antecedentes, como doenças, prática desportiva e acidentes.

\section{Organização e descrição das categorias das variáveis}

A fim de facilitar a análise e apresentação dos resultados, as categorias ou subdivisões das variáveis foram agrupadas em blocos mais expressivos de resultados, sem, no entanto, modificar a essência de sua origem ou as conclusões do estudo.

Os tipos de trabalho foram divididos em duas categorias: atividades manuais e serviços gerais, segundo a característica ocupacional. Foram 
consideradas atividades manuais aquelas em que as participantes executavam gestos repetitivos e ficavam sentadas na maior parte do tempo, realizando trabalhos como confecção de bijouterias, colares e bandeirinhas para festas, arremate, costura, empacotamento de alho, embalagens, digitação, catalogação de livros e produtos de consumo. Foram considerados serviços gerais aqueles nos quais as reeducandas despendiam maior gasto energético e permaneciam mais tempo em pé ou agachadas, como faxina geral, cozinha, lavanderia, horta, enfermaria e clic (reeducandas que abrem e fecham portões para deslocamento de pessoas).

As regiões anatômicas foram agrupadas em membros superiores, membros inferiores e coluna. Por fim, o tempo de trabalho foi expresso em meses, sendo dividido em três grupos: (i) de 0 a 5, (ii) de 6 a 11 e (iii) 12 ou mais. Cabe destacar que o tempo de trabalho e reclusão têm relação direta, pois, assim que inseridas no Centro de Ressocialização, as detentas iniciam suas ocupações laborais.

\section{Procedimentos estatísticos}

Os valores descritivos foram apresentados na forma de distribuição de freqüências ou taxas. A comparação de ocorrência de queixa osteomuscular e suas características entre variáveis antropométricas foi realizada pelos testes t de Student, teste não paramétrico de Mann Whitney, teste não paramétrico de Kruskal-Wallis e teste paramétrico por análise de variância para o modelo com um fator, complementado com o teste de compassos múltiplos de Tukey.

Para o estudo da associação entre características das queixas e região anatômica ou tempo de trabalho, foi utilizado o teste de Goodman para associação entre populações multinomiais e dentro delas 13. Todas as discussões dos resul- tados foram realizadas para nível de 5\% de significância.

\section{Aspectos legais da pesquisa}

A pesquisa foi iniciada após aprovação pelo Comitê de Ética em Pesquisa da Faculdade de Medicina de São José do Rio Preto e autorização pelos órgãos competentes que gerenciam a unidade prisional (Diretoria Geral do Centro de Ressocialização Feminino de São José do Rio Preto e Grupo de Amparo ao Doente de AIDS - GADA). A participação da população ocorreu mediante leitura, compreensão e autorização por escrito de um Termo de Consentimento Livre e Esclarecido.

\section{Resultados}

Após a exclusão das mulheres com antecedentes de queixas músculo-esqueléticos, o total do universo possível para coleta resultou em 155 sujeitos. Dessa amostra, 146 reeducandas $(94,19 \%)$ passaram a referi-las após inicio de atividade laboral e representam o objeto de estudo da pesquisa.

Na Tabela 1, são apresentados valores absolutos e relativos de acometidas e de queixas referidas, além de um modelo descritivo de correção, representado pela razão entre queixas e participantes. Verificou-se que as taxas de queixas foram próximas nas categorias ocupacionais.

A exploração entre variáveis antropométricas e nível de severidade da queixa é apresentada na Tabela 2. Não houve diferença estatística entre os níveis para idade e estatura, todavia observou-se que maiores valores de peso associaram-se com os níveis moderado e acentuado, apresentando diferença significante em relação aos menores valores de peso, que se associaram às queixas consideradas de nível leve.

Distribuição das participantes acometidas, freqüência de queixas e taxas de queixas segundo ocupação.

\begin{tabular}{|c|c|c|c|c|c|}
\hline \multirow[t]{2}{*}{ Ocupação } & \multicolumn{2}{|c|}{ Total de indivíduos } & \multicolumn{2}{|c|}{ Total de queixas } & \multirow{2}{*}{$\begin{array}{c}\text { Taxa de queixa por } \\
\text { participante acometida * }\end{array}$} \\
\hline & $\mathbf{n}$ & $\%$ & $\mathbf{n}$ & $\%$ & \\
\hline Atividades manuais & 117 & 80,14 & 324 & 80,40 & 2,77 \\
\hline Serviços gerais & 29 & 19,86 & 79 & 19,60 & 2,72 \\
\hline Total & 146 & 100,00 & 403 & 100,00 & 2,76 \\
\hline
\end{tabular}

* A taxa de queixa por participante acometida é obtida pela divisão do número total de queixas pelo número total de reeducandas entrevistadas em cada ocupação. 
Em relação às variáveis antropométricas e necessidade de afastamento, notou-se que as médias de idade e peso são maiores entre as participantes com necessidade de afastamento, apresentando diferença significante em relação às médias das mesmas variáveis para as que não foram afastadas em virtude de queixas relacionadas ao trabalho. Para a variável estatura, não foi notada diferença entre os grupos analisados. Tais achados são apresentados na Tabela 3 .

Observa-se, na Tabela 4, a distribuição dos relatos de nível de severidade em relação aos locais acometidos. Para todas as regiões anatômicas, as queixas acentuadas foram mais referidas quando comparadas às moderadas e leves $(p<0,05)$. Para agravos da coluna do tronco, observou-se que os relatos de queixa acentuada foram mais referidos quando comparados aos relatos sobre membros superiores e inferiores $(\mathrm{p}<0,05)$.
Na Tabela 5, são apresentados os resultados da associação entre necessidade de afastamento e tempo de trabalho. Observou-se que o maior tempo de exposição ao trabalho, seis meses ou mais, caracteriza risco para condições que levam ao afastamento $(\mathrm{p}<0,05)$

\section{Discussão}

Na revisão de literatura para elaboração deste estudo, notou-se que os problemas ou distúrbios músculo-esqueléticos relacionados ao trabalho são relatados desde o ano de 1700 , com o sofrimento de artesãos escriturários, passando pela revolução industrial, com o trabalho automatizado e repetitivo, que visava ao aumento de produtividade, sem que houvesse autocontrole sobre o ritmo, posição e modo de trabalho, bem como mobiliário e equipamentos ergonomicamente

Tabela 2

Medidas descritivas das participantes da pesquisa segundo a severidade da queixa.

\begin{tabular}{lccccc}
\hline Variável & $\begin{array}{c}\text { Medida } \\
\text { descritiva }\end{array}$ & Leve & $\begin{array}{c}\text { Severidade da queixa } \\
\text { Moderada }\end{array}$ & Acentuada \\
\hline Idade (anos) * & Mediana & 31 & 26 & 29 & Valor de $p$ \\
& Média & $32,5 \pm 9,4$ & $29,9 \pm 10,4$ & $31,7 \pm 9,5$ & 71,0 \\
Peso $(\mathrm{kg}) * *$ & Mediana & 63,0 & 67,5 & $73,4 \pm 16,1 \mathrm{~b}$ & 160,0 \\
& Média & $64,5 \pm 12,0 \mathrm{a}$ & $71,0 \pm 15,7 \mathrm{~b}$ & 16,0001 & $>0,05$ \\
Estatura $(\mathrm{cm}) * *$ & Mediana & 160,0 & 162,0 & $169,9 \pm 6,8$
\end{tabular}

Nota: Letras minúsculas indicam comparação de grupos, em cada coluna (severidade da queixa), fixada a categoria de resposta, considerando a $<$ b.

* Teste estatístico realizado com base nos valores da mediana;

** Teste estatístico realizado com base nos valores da média e desvio-padrão.

Tabela 3

Medidas descritivas das participantes da pesquisa segundo necessidade de afastamento.

\begin{tabular}{|c|c|c|c|c|}
\hline \multirow[t]{2}{*}{ Variável } & \multirow{2}{*}{$\begin{array}{c}\text { Medida } \\
\text { descritiva }\end{array}$} & \multicolumn{2}{|c|}{ Necessidade de afastamento } & \multirow[t]{2}{*}{ Valor de $\mathrm{p}$} \\
\hline & & Não & $\operatorname{Sim}$ & \\
\hline \multirow[t]{2}{*}{ Idade (anos) * } & Mediana & 25 & 31 & $<0,0001$ \\
\hline & Média & $27,9 \pm 7,9$ & $32,9 \pm 10,2$ & \\
\hline \multirow[t]{2}{*}{ Peso $(\mathrm{kg}) * \star$} & Mediana & 63,0 & 70,5 & $<0,0001$ \\
\hline & Média & $66,4 \pm 13,4$ & $73,1 \pm 16,1$ & \\
\hline \multirow[t]{2}{*}{ Estatura $(\mathrm{cm}) \star \star$} & Mediana & 160,0 & 160,0 & $>0,05$ \\
\hline & Média & $161,1 \pm 7,2$ & $159,8 \pm 6,1$ & \\
\hline
\end{tabular}

* Teste estatístico realizado com base nos valores da mediana;

** Teste estatístico realizado com base nos valores da média e desvio-padrão. 
Distribuição dos relatos do nível de dor em relação à região anatômica acometida.

\begin{tabular}{|c|c|c|c|c|}
\hline \multirow[t]{2}{*}{ Região anatômica } & \multicolumn{4}{|c|}{ Severidade da queixa } \\
\hline & Leve & Moderada & Acentuada & Total \\
\hline \multirow[t]{2}{*}{ Coluna } & $24(12,70) a$ & $56(29,63) a$ & $109(57,67) b$ & 189 \\
\hline & A & $\mathrm{B}$ & C & \\
\hline \multirow[t]{2}{*}{ Membros superiores } & $29(23,39) b$ & $38(30,65) a$ & $57(45,97) a$ & 124 \\
\hline & $A$ & A & B & \\
\hline \multirow[t]{2}{*}{ Membros inferiores } & $25(27,78) b$ & $24(26,67) a$ & $41(45,56) a$ & 90 \\
\hline & $A$ & $A$ & B & \\
\hline
\end{tabular}

Nota: Dados analisados com base no teste de Goodman, com conclusões consideradas para p < 0,05. Para comparação entre os valores, são utilizadas letras. Quando há diferença estatística significante, as letras são diferentes; quando não há, são iguais. Letras minúsculas indicam comparação de grupos, em cada coluna (severidade da queixa), fixada a categoria de resposta, considerando $a<b$. Letras maiúsculas indicam comparação de categorias de resposta, em cada linha (região anatômica), dentro do grupo, considerando $\mathrm{A}<\mathrm{B}<\mathrm{C}$.

adequados, como observados atualmente, conforme descreveram Ribeiro 2 e Walsh et al. 4 .

A escolha do tema para realização da presente pesquisa baseou-se não só no contexto histórico descrito, mas também nas freqüentes queixas osteomusculares das reeducandas à equipe se saúde da unidade prisional em questão. De fato, a elevada ocorrência de relatos dessa natureza foi notada nos achados do presente estudo e corrobora informações de pesquisadores que investigaram o assunto 7,14,15,16,17.

Do ponto de vista etiológico, nota-se que a maior freqüência desses agravos acomete mulheres jovens, na faixa etária entre 20 e 39 anos, segundo relatam Walsh et al. ${ }^{4}$ e Reis et al. 17. Essas características são semelhantes às das participantes deste estudo, resultando em excelente condição de controle para esta investigação.

Especificamente sobre o método utilizado para a coleta das informações sobre queixas osteomusculares relacionadas ao trabalho, foi utilizado, como base, o NMQ descrito por Kuoringa et al. 12, que foi desenvolvido com a proposta de padronizar resultados e facilitar a interpretação de achados entre estudos. Pinheiro et al. ${ }^{6}$ sugeriram modificações para aplicações futuras do NMQ, como o grau de severidade das queixas e registros anteriores às atividades ocupacionais, como acidentes, doenças ou práticas esportivas, mudanças que foram incorporadas neste estudo. Acrescentou-se, também, ao desenho da figura humana que ilustrava o questionário, independentemente de sugestões anteriores, as regiões anatômicas do braço e panturrilha, referidas pelas participantes durante estudo piloto. Destaca-se que as alterações realizadas contribuíram
Tabela 5

Distribuição dos relatos de necessidade de afastamento em relação ao tempo de trabalho.

\begin{tabular}{lccc}
\hline Tempo & Não & Afastamento & Total \\
\hline Até 5 meses & $56(45,90) b$ & $66(54,10) \mathrm{b}$ & 122 \\
De 6 a11 meses & A & B & 181 \\
1 ano ou mais & $41(22,65) \mathrm{a}$ & $140(77,35) \mathrm{b}$ & 100 \\
& A & B & \\
\hline
\end{tabular}

Nota: Dados analisados com base no teste de Goodman, com conclusões consideradas para $\mathrm{p}<0,05$. Para comparação entre os valores, são utilizadas letras. Quando há diferença estatística significativa, as letras são diferentes; quando não há, são iguais. Letras minúsculas indicam comparação de grupos, em cada coluna (afastamento), fixada a categoria de resposta, considerando $\mathrm{a}<\mathrm{b}$. Letras maiúsculas indicam comparação de categorias de resposta, em cada linha (tempo), dentro do grupo, considerando $A<B$.

de forma importante para seleção da amostra e análise da associação entre variáveis.

Apesar de o questionário ser auto-aplicável, como sugeriram Barros \& Alexandre 11, a coleta dos dados foi realizada pelo pesquisador, por entender que haveria menos vieses em relação à compreensão das questões, já que as respondentes tinham diferentes graus de instrução e, ainda, conforme descreveram Miranda et al. 10 em estudo com população da mesma natureza, baixos níveis sócio-econômicos e educacionais.

Sobre a população, dentre as incluídas para participar do desenho metodológico proposto, $94,19 \%$ referiram queixas osteoarticulares rela- 
cionados às atividades ocupacionais. Tal registro é semelhante aos achados de Gurgueira et al. 5, que observaram elevada freqüência de agravos relacionados ao trabalho em seu estudo.

As taxas de queixas encontradas nas categorias de trabalho definidas como serviços gerais e trabalhos manuais foram próximas. Como hipótese explicativa, Reis et al. 17 e Lin et al. 18 descrevem que, independentemente do tipo de atividade, ao existir repetitividade gestual, posição inadequada, impactos, vibrações e pressão mecânica sobre determinado local do corpo, há exposição a fatores de risco para doenças osteoarticulares, concordando com os achados quantitativos desta pesquisa.

Observou-se que, para a variável peso, a necessidade de afastamento pode estar relacionada à severidade das queixas. De fato, Crill \& Hoster 19, explorando causas de dor nas costas em auxiliares de emergência, destacaram o sobrepeso como um dos fatores para instalação de lesões. Do ponto de vista biomecânico, entendese que tal fato é associado à sobrecarga funcional de estruturas do corpo, conforme descreveram Murphy et al. 20 em estudo de revisão. Os autores consideram o aumento de peso como fator de risco para instalação de agravos em praticantes de atividades motoras, pela proporcional resultante de força em articulações, tendões e músculos.

Notou-se que, para as participantes afastadas, os valores para idade foram significativamente maiores do que os achados para as que não necessitaram de afastamento. Apesar do descrito, não se pode afirmar que idades avançadas são determinantes nas ocorrências de DORT, já que o valor da mediana de idade para o grupo acometido foi de 31 anos. Todavia, essa faixa de idade, que representa a fração de alta produtividade de trabalho da sociedade, também foi observada nos estudos de Walsh et al. 4 e Reis et al. 17 .

Embora as queixas acentuadas tenham sido mais referidas do que as moderadas e leves em todas as regiões anatômicas, a coluna do tronco se destacou quando comparada aos membros superiores e inferiores. Tal condição pode estar associada a situações ergonômicas inadequadas, incluindo fadiga pela repetição de gestos e má postura durante a realização de tarefas laborais, conforme sugerem Brandão et al. ${ }^{14}$. Além disso, a coluna vertebral é descrita como local de alta prevalência para instalação de lesões relacionadas ao trabalho e enfatizada nas atividades laborais em indivíduos que as executam sentados por longos períodos, resultando em pressão dos discos intervertebrais, o que constitui fator de risco para dores nessa região 7,21 .

A necessidade de afastamento das atividades ocupacionais foi mais evidenciada nas re- educandas que trabalhavam há mais tempo do que as que não foram afastadas. Nesse sentido, mesmo não explorando a variável relacionada ao afastamento de trabalho, Forde et al. 22 comentam que, com o maior volume de exposição à atividade laboral, aumentam as possibilidades de queixas, com conseqüente necessidade de afastamento.

É observado na prática que, devido ao fato de que a cada três dias trabalhados desconta-se um dia da pena a ser cumprida, as reeducandas fazem opção de trabalhar, mesmo na presença de queixas. Contudo, a partir da continuação das atividades, movimentos repetitivos e possível postura incorreta, as queixas podem se acentuar, diminuindo a produtividade e, por fim, resultar em impossibilidade de exercer suas funções, levando ao afastamento, o que está de acordo com o estudo realizado por Brandão et al. 14 em bancários da Região Sul do Brasil. Essa condição parece ser realidade na população brasileira em geral, ou seja, insistência pela continuação de atividades mesmo diante da presença de problemas físicos, mas, nesse caso, pela necessidade sócio-econômica de emprego para subsistência.

\section{Considerações finais}

Como limitação do estudo, pode-se destacar a ausência de informações sobre a prática de atividades motoras das entrevistadas anterior à reclusão. Tais características poderiam complementar os resultados deste estudo de modo a associá-las à presença ou não de queixas osteomusculares. No entanto, favorecendo o efeito controle, destaca-se que, após a inserção no Centro de Ressocialização Feminino de São José do Rio Preto, as reeducandas não eram submetidas a quaisquer programas de atividade física.

Com base nos resultados obtidos neste estudo, verificou-se alta freqüência de queixas osteomusculares relacionadas ao trabalho na população estudada, bem como associação de sua ocorrência e severidade às variáveis antropométricas e características laborais. Esses achados devem incentivar a elaboração de planos de ação preventivos e terapêuticos específicos às causas, para amenizar as queixas e contribuir com a qualidade de trabalho e de vida da população estudada.

Programas sistematizados de atividades físicas orientadas e ou de ginástica laboral supervisionada poderiam contribuir para minimizar os problemas descritos, já que tais práticas auxiliam no equilíbrio das funções motoras, como manutenção de bons níveis de tônus muscular, 
amplitude e estabilidade articular, além de potencializar um melhor funcionamento neurohumoral, conforme descrito amplamente no meio científico.

\section{Resumo}

Considerando as queixas de origem laboral um problema de saúde pública, objetivou-se, com o presente estudo, analisar a freqüência da população que refere queixas osteomusculares e a associação de ocorrência e severidade destas às variáveis antropométricas e de trabalho. Tomou-se 146 mulheres de um centro de ressocialização feminino, que responderam a um questionário validado contendo informações sobre dados antropométricos e queixas osteomusculares relacionadas ao trabalho. O estudo da associação entre e dentro das variáveis foi feito pelo teste de Goodman. Observou-se elevada freqüência de ocorrência de queixas após início de atividade laboral (94,19\%). As participantes com necessidade de afastamento apresentaram maiores valores em idade e peso. Queixa acentuada foi mais referida na coluna do tronco. Afastamento foi mais referido para as que trabalhavam há mais tempo. Concluiu-se que é alta a freqüência de queixas relacionadas ao trabalho e que há associação entre maiores valores de peso e de estatura e nível de severidade acentuado; maiores valores de idade e de peso e necessidade de afastamento; níveis acentuados de queixas e região da coluna e entre maior tempo de serviço e necessidade de afastamento.

Transtornos Traumáticos Cumulativos; Saúde da Mulher; Saúde Ocupacional; Antropometria
Em adição, parece adequado investigar populações diversas valendo-se da metodologia proposta neste trabalho, a fim de garantir a especificidade de ações a cada tipo de ocupação que apresente fatores para instalação das DORT.

\section{Colaboradores}

E. C. Pastre, G. Carvalho Filho e C. M. Pastre participaram integralmente de todas as fases do trabalho, desde o desenho do estudo à redação final. C. R. Padovani colaborou no desenho do estudo, na elaboração do modelo estatístico e na discussão dos achados. J. S. Almeida e J. Netto Júnior participaram do levantamento e seleção de literatura, tabulação dos dados e da discussão dos achados. 


\section{Referências}

1. Merlo ARC, Jacques MGC, Hoefel MGL. Trabalho de grupo com portadores de LER/DORT: relato de experiência. Psicol Reflex Crít 2001; 14:253-8.

2. Ribeiro HP. Lesões por Esforços Repetitivos (LER): uma doença emblemática. Cad Saúde Pública 1997;13 Suppl 2:S85-93.

3. Takahashi MABC, Canesqui AM. Pesquisa avaliativa em reabilitação profissional: a efetividade de um serviço em desconstrução. Cad Saúde Pública 2003; 19:1473-83.

4. Walsh IAP, Corral S, Franco RN, Canetti MER, Coury HJCG. Capacidade para o trabalho em indivíduos com lesões músculo-esqueléticas crônicas. Rev Saúde Pública 2004; 38:149-56.

5. Gurgueira GP, Alexandre NMC, Corrêa Filho HR. Prevalência de sinais e sintomas músculo-esqueléticos em trabalhadoras de enfermagem. Rev Latinoam Enfermagem 2003; 11:608-13.

6. Pinheiro FA, Tróccoli BT, Carvalho CV. Validação do Questionário Nórdico de Sintomas Osteomusculares como medida de morbidade. Rev Saúde Pública 2002; 36:307-12.

7. Santos Filho SB, Barreto SM. Atividade ocupacional e prevalência de dor osteomuscular em cirurgiões-dentistas de Belo Horizonte, Minas Gerais, Brasil: contribuição ao debate sobre os distúrbios osteomusculares relacionados ao trabalho. Cad Saúde Pública 2001; 17:181-93.

8. Decreto no. 48.802, de 21 de julho de 2004. Diário Oficial do Estado de São Paulo 2004; 22 jul.

9. Pereira MG. Epidemiologia: teoria e prática. Rio de Janeiro: Editora Guanabara Koogan; 1995.

10. Miranda AEM, Vargas PRM, Viana MC. Saúde sexual e reprodutiva em penitenciária, Espírito Santo, Brasil. Rev Saúde Pública 2004; 38:255-60.

11. Barros EN, Alexandre NM. Cross-cultural adaptation of the Nordic musculoskeletal questionnaire. Int Nurs Rev 2003; 50:101-8.

12. Kuoringa I, Jonsson B, Kilbom A, Vinterberg H, Biering-Sorensen F, Andersson G, et al. Standardised Nordic questionnaires for the analysis of musculoskeletal symptoms. Appl Ergon 1987; 18:233-7.
13. Campana AO, Padovani CR, Timo-Iara C, Freitas CBD, Paiva SAR, Hossne WS. Investigação científica na área médica. São Paulo: Editora Manole; 2001.

14. Brandão AG, Horta BL, Tomasi E. Sintomas de distúrbios osteomusculares em bancários de Pelotas e região: prevalência e fatores associados. Rev Bras Epidemiol 2005; 8:295-305.

15. Kivimãki M, Vahtera J, Ferrie JE, Hemingway $H$, Pentti J. Organisational downsizing and musculoskeletal problems in employees: a prospective study. Occup Environ Med 2001; 58:811-7.

16. Leclerc A, Chastang J-F, Niedhammer I, Landre M-F, Roquelaure Y; Study Group on Repetitive Work. Incidence of shoulder pain in repetitive work. Occup Environ Med 2004; 61:39-44.

17. Reis RJ, Pinheiro TMM, Navarro A, Martin MM. Perfil da demanda atendida em ambulatório de doenças profissionais e a presença de lesões por esforços repetitivos. Rev Saúde Pública 2000; 34:292-8.

18. Yeng LT, Teixeira MJ, Romano MA, Picarelli $\mathrm{H}$, Settimi MM, Greve JMD. Distúrbios ósteo-musculares relacionados ao trabalho. Rev Méd (São Paulo) $2001 ; 80: 422-42$.

19. Cril MT, Hoster D. Back strength and flexibility of SEM providers in practicing prehospital providers. J Occup Rehabil 2005; 15:105-11.

20. Murphy DF, Connolly DA, Beynnon BD. Risk factors for lower extremity injury: a review of the literature. Br J Sports Med 2003; 37:13-29.

21. McGeary DD, Mayer TG, Gatchel RJ, Anagnostis C, Proctor TJ. Gender-related differences in treatment outcomes for patients with musculoskeletal disorders. Spine J 2003; 3:197-203.

22. Forde MS, Punnet L, Wegman DH. Prevalence of musculoskeletal disorders in union ironworkers. J Occup Environ Hyg 2005; 2:2003-12.

Recebido em 06/Abr/2006

Versão final reapresentada em 15/Jan/2007 Aprovado em 19/Abr/2007 